$$
\begin{aligned}
& 0.94-950226-51 \\
& \text { UCRL-IC-120177 } \\
& \text { PREPRINT }
\end{aligned}
$$

\title{
Modeling of Ablation by Photospallation Using the Computer Program PUFF/DFRACT
}

\author{
Tarabay Antoun*, Lynn Seaman*, \\ and Michael E. Glinsky
}

This paper was prepared for submittal to the SPIE's 1995 International Symposium on Lasers and Applications/Biomedical Optics

Laser-Tissue Interaction VI, San Jose, CA

February 4-10, 1995

March 1, 1995

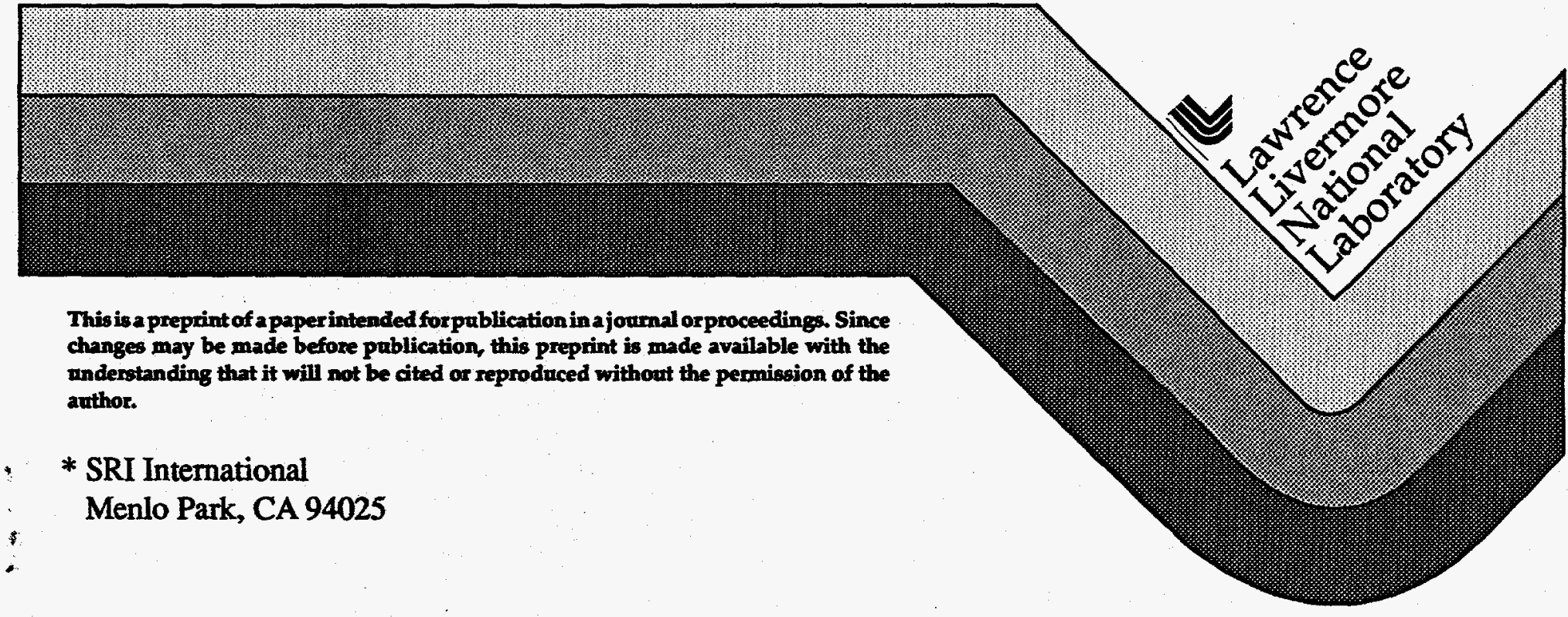

DistRIBUTION OF THIS DOCUMENT IS UNLIMITED BS MAS 


\section{DISCLAMMER}

This document was prepared as an account of work sponsored by an agency of the United States Government. Neither the United States Government nor the University of California nor any of their employees, makes any warranty, express or implied, or assumes any legal liability or responsibility for the accuracy, completeness, or usefulness of any information, apparatus, product, or process disclosed, or represents that its use would not infringe privately owned rights. Reference herein to any specific commercial product, process, or service by trade name, trademark, manufacturer, or otherwise, does not necessarily constitute or imply its endorsement, recommendation, or favoring by the United States Government or the University of California. The views and opinions of authors expressed herein do not necessarily state or reflect those of the United States Government or the University of California, and shall not be used for advertising or product endorsement purposes. 


\section{DISCLAIMER}

Portions of this document may be illegible in electronic image products. Images are produced from the best available original document. 


\title{
Modeling of ablation by photospallation using the computer program PUFF/DFRACT
}

\author{
Tarabay Antoun and Lynn Seaman \\ SRI International \\ Menlo Park, California 94025 \\ Michael E. Glinsky \\ Lawrence Livermore National Laboratory \\ Livermore, California 94550
}

\section{ABSTRACT}

In general, macroscopic material failure is a manifestation of irreversible changes at the microscopic level. Many tissues, which may appear to be macroscopically homogeneous, are, at a fundamental microscopic level, a composite material. For example, cornea is composed of a hyaluronic acid matrix in which layers of collagen fibers are overlaid in a crossing pattern. The points where the collagen fibers intersect are potential nucleation sites for microscopic defects, which under the action of tensile stress, nucleate, grow and coalesce to form macroscopic failure planes, or spall planes. Using a model based on microstructural evolution, this paper examines the failure process during photoablation. Specifically, the paper describes a physically motivated, micromechanical model based on the nucleation and growth of spherical voids. This model is then used to simulate photoablation of cornea. Potential for using this model to predict the stress wave and material damage measured by experiment is discussed.

Keywords: laser, photospallation, ablation, tissue

\section{INTRODUCTION}

For short pulse laser ablation, where the sound transient time across the zone of energy deposition is greater than the pulse length, a stress wave is launched into the tissue. The magnitude of the peak stress and the shape of the stress transient generated during deposition depends on the laser pulse characteristics (fluence, duration, etc.) and on the properties of the tissue (absorption depth, sound speed, etc.). The mechanical response of the tissue may vary from linear elastic at small strains to nonlinear and inelastic at larger strains. The nonlinearity in the stress-strain relation is due to material damage which is a manifestation of irreversible changes that take place at a microscopic level. This damage begins to evolve when the tissue is exposed to stress waves of sufficient magnitude and duration to destroy the microscopic structure of the material. Support structures, analogous to reinforcing steel in concrete, can be broken and voids can be generated. The voids grow under the action of tensile stresses thereby decreasing the ability of the material to support shear and tensile stresses. In the limiting case of no material strength, one is left with only hydrodynamic motion. The microscopic damage therefore has a deterministic effect on the macroscopic propagation of the stress waves.

The near-instantaneous deposition of laser energy into tissue leads to thermal stresses because, locally, the material is heated faster than it can expand. Since the energy deposition profile is not uniform throughout the depth of the tissue, the resulting stress profile is not uniform and the material is in a state of non-equilibrium. But, materials, in general, cannot sustain discontinuities (i.e., non-equilibrium) in either stress or particle velocity. As a result, compressive stress waves are produced to carry the discontinuities away and leave the material in a state of equilibrium. These stress waves originate in, and propagate away from the energy deposition region both towards the interior and free surface of the tissue. Upon reaching the free surface, the compressive stress wave reflects back into the tissue as a tensile wave and a bipolar stress transient develops and propagates towards the interior of the tissue. Since most materials fail in tension before they fail in compression, the magnitude of the tensile component of the wave is limited by the ability of the material to support tensile loads. The maximum amount of damage occurs some distance into the material, the distance being determined by the material properties, the laser absorption length and the laser fluence. The material will cleave at this layer identified as the spall plane. The chipping off of the surface by the propagation of a laser induced stress wave is called photospallation. ${ }^{1}$ For a large laser 
fluence it should be noted that the material can lose its integrity not only near the spall plane but over a large volume in the deposition region near the surface. This volume will expand hydrodynamically in a process that is commonly called explosive vaporization.

Many biological tissues are complex composite materials. For example, the cornea is made up of a hyaluronic acid matrix in which layers of collagen fibers are overlaid in a crossing pattern (Fig. 1). The fibers are laid out in a mesh with the orientation of each fiber layer rotated by $90^{\circ}$ with respect to the orientation of the overlying layer (see Fig. 1). The fibers are about $50 \mathrm{~nm}$ in diameter and fill $1 / 5$ of the volume. ${ }^{2}$ The interstitial volume is filled by hyaluronic acid whose viscosity varies depending on strain rates. It has 1000 times the viscosity of water at low stain rates but its viscosity decreases to that of water at strain rates of $10^{6} \mathrm{~Hz}^{3}$ The points where the collagen fibers cross serve as sites for nucleation of voids. This structure is similar to a reinforced concrete where the collagen fibers are the "reinforcing bars" and the hyaluronic acid is the "concrete". Another interesting effect that will be discussed later is that the strength of the collagen fibers can be greatly reduced by heating. This phenomenon, known as "thermal softening" is known to occur in most solids where the yield strength varies from some initial value at room temperature to zero at or near the melting temperature.

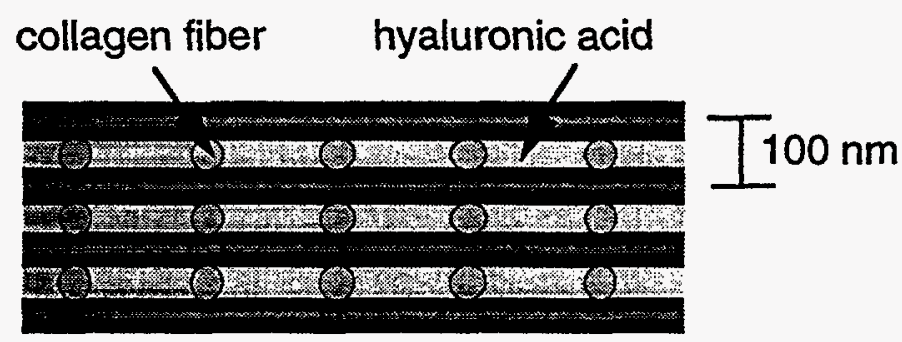

Fig. 1. Cornea structure showing matrix of collagen fibers embedded in hyaluronic acid.

The material failure process is of both direct and indirect interest. It is of direct interest when one considers photoacoustic drug delivery. The fractures that are the primary cause of the material failure increase the surface area of the material perhaps leading to greater drug penetration into tissue and faster drug absorption. ${ }^{4}$ An example of such delivery would be the fracturing of a thrombus to allow greater penetration of urokinase for thrombolysis. One could also be interested in the damage to cellular structures caused by the stress wave with an eye cast towards minimizing this damage.

There are several macroscopic phenomena that are an indirect result of the microscopic damage: ablation threshold and the stress wave propagated into the bulk tissue. An elegant experiment ${ }^{5}$ was done showing the difference between delivering the energy to the structural "rebar" of the tissue (extra cellular matrix, that is, ECM) and delivering it to the interstitial "concrete" or the tissue (water). Delivery to the ECM (with a UV laser) showed a much lower ablation threshold and a simple bipolar stress wave with the tensile part truncated at the spall strength of water. Delivery to the water (IR laser) showed a higher ablation threshold and an interesting double humped compressive stress wave for a laser fluence modestly above the threshold. It is surmised that the difference is due to the ECM being destroyed in one case and not the other. There is also a possibility that the material is being thermally softened at later times when the energy is delivered to the water. This could be due to the energy diffusing into the ECM, gradually destroying its structural integrity. Although simple models, vaporization and material blowoff, explain the ablation threshold for the UV and IR lasers, respectively, a more detailed model is needed to predict the stress waves. The two simple models are really limiting cases of the more general case and details of the ablation in the transition between the these two cases may be the source of the interesting pulse form for IR laser ablation. In addition a more sophisticated model is required to make predictions of the size of the ejected particles or details of the microscopic damage (such as void density and size). Experimental information such as this is available in transparent material via optical flash photography. ${ }^{6}$ The photographs show the evolution of the bubbles or voids in the liquid or tissue.

A model for this microscopic damage and how it influences the macroscopic stress wave propagation will be presented in the next section. It will be applied to a case similar in some respects to cornea ablation. How these results could be used and could be compared to experiment will be discussed. 


\section{MODEL}

A one-dimensional, finite difference, wave propagation hydrocode, SRI PUFF 8, is used to simulate the response of cornea tissue during laser ablation. The mechanical behavior of the tissue is modeled using a ductile fracture model originally developed for metal failure, DFRACT (an acronym for Ductile FRACTure). ${ }^{7}$ DFRACT is based on the nucleation and growth of microscopic voids, and the manner in which they affect the strength and subsequent behavior of the material. The model assumes a microstatistical void distribution of the form

$$
N(R)=\bar{N} \exp (-R / \bar{R})
$$

where $\bar{N}$ is the total number of voids (per unit volume) greater than $\mathrm{R}$ and $\bar{R}$ is the void size that characterizes the distribution. The evolution of damage in terms of the number of voids per unit volume and void size is monitored in the model with two rate equations that depend on the yield strength, the number of nucleation sites (taken equal to the number of intersection point of the collagen fibers), the initial flaw size (taken equal to the size of the collagen fiber), and the viscosity of the material (taken equal to the viscosity of hyaluronic acid). An example of how the void size distribution varies with time is shown in Fig. 2.

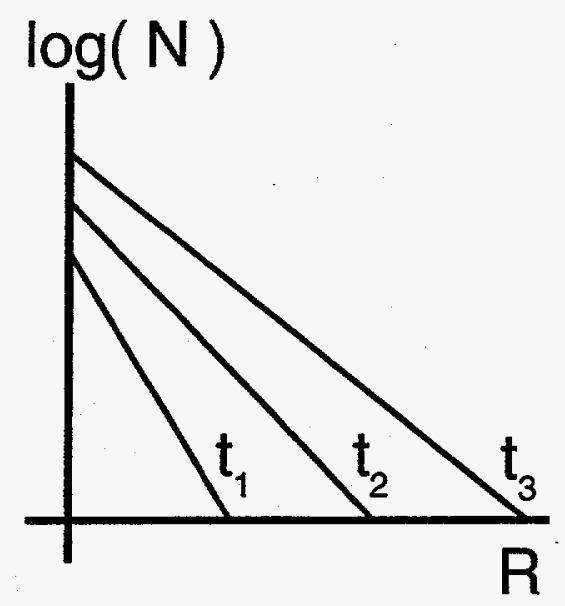

Fig. 2. Example of how void distribution function in one cell of the simulation varies with time $\left(t_{1}<t_{2}<t_{3}\right)$.

The laser energy is deposited according to a Beer-Lambert law, $I=I_{o} e^{-\mu x}$, valid for a highly absorbing tissue. Here, $I_{o}=35 \mathrm{~mJ} / \mathrm{cm}^{2}$ is the laser fluence, $2 \mathrm{~ns}$ is the pulse length, and $\mu=2700 / \mathrm{cm}$ is the absorption coefficient. The laser is incident on a front free surface of a tissue which has no back surface. A Mie-Grüniesen EOS is used with a $\Gamma=0.15$ consistent with that of water. The spall strength is taken to be that of water $10^{6}$ dynes $/ \mathrm{cm}^{2}$, the initial flaw size is $50 \mathrm{~nm}$ and the number of nucleation sites is equal the number of fiber crossings $3 \times 10^{14} / \mathrm{cc}$.

\section{RESULTS}

The results of the simulation are shown in Fig. 3. Note the small magnitude of the tensile component of the stress wave as compared with the compressive component. This is due to the evolution of damage in the form of void growth under tension, which in turn limits the ability of the material to carry the load. Consequently, the tensile strength of the material is limited to the spall strength of water. The decrease in the peak compressive stress depicted in the figure is due to the dispersion of the wave as it propagates into the material, not due to yielding or damage under compression.

The plot of void volume fraction as a function of depth shows the development of a spall plane at $2 \mu \mathrm{m}$ where the percentage void volume reaches a maximum, with a damaged zone extending from the free surface to a depth of about $5 \mu \mathrm{m}$. 
As expected, the highest temperatures are reached in the energy deposition region. The maximum temperature reached during the simulation occurred at the front free surface and was equal to about $56{ }^{\circ} \mathrm{C}$. Substantially lower peak temperatures were reached in the region behind the spall plane.
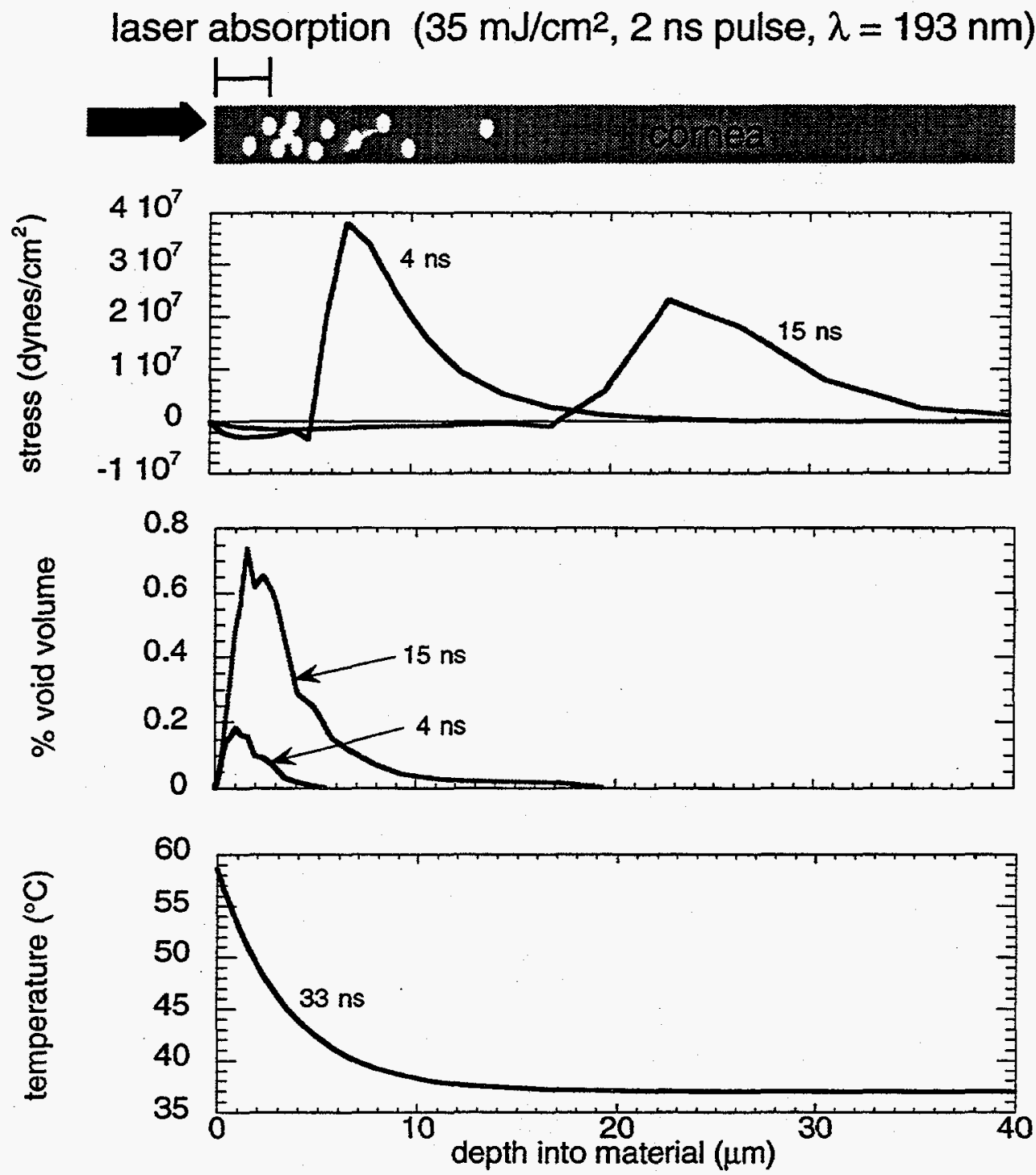

Fig. 3. Results of simulation.

\section{DISCUSSIONS AND CONCLUSIONS}

This method of analysis has the ability to predict the stress wave that travels into the tissue as well as the density and size of the voids. All of these are measurable quantities. To compare to a case such as the $\mathbb{R}$ ablation of dermis, ${ }^{5}$ one needs to increase the yield strength in our simulation to be equal to that of the ECM. In addition one would add a temperature and time dependent reduction of the yield strength to be consistent with the destruction of the structural integrity of the ECM. The challenge is then to reproduce not only the measured maximum stress vs. laser fluence but the behavior of the stress pulses at various laser fluences. This will be the subject of future work. 


\section{ACKNOWLEDGMENTS}

This work was performed under the auspices of the U.S. Department of Energy by the Lawrence Livermore National Laboratory under contract W-7405-ENG-48.

\section{REFERENCES}

1. R.S. Dingus and R. J. Scammon, "Grüniesen-stress induced ablation of biological tissue," Proceedings of LaserTissue Interaction II, Vol. 1427, pp. 45-54, 1991.

2. D.M. Maurice, "The Cornea and Sclera," The Eye, Vol. $1,2^{\text {nd }}$ edition, edited by Hugh Davison, Academic Press, New York, 1961.

3. Y.C. Fung, Biomechanics: Mechanical Properties of Living Tissues, $2^{\text {nd }}$ edition, Springer-Verlag, New York, 1993.

4. H. Shangguan, A Shearin, K.W. Gregory and S.A. Prahl, "Photoacoustic drug delivery: effect of laser parameters on spatial distribution of delivered drug," Proceedings of Laser-Tissue Interaction VI, Vol. 2391, in press, 1995.

5. V. Venugopalan, "Pulsed-laser ablation: rapid surface vaporization or thermal explosion?", Proceedings of LaserTissue Interaction VI, Vol. 2391, in press, 1995.

6. G. Paultauf, E. Reichel and H. Schmidt-Kloiber, "Study of different ablation models by use of high-speed-sampling photography," Proceedings of Laser-Tissue Interaction III, Vol. 1646, pp. 343-352, 1992.; G Paltauf and H Schmidt-Koiber, "Investigation of ablation dynamics as a function of wavelength", Proceedings of Laser-Tissue Interaction V, Vol. 2134, pp. 93-103, 1994; R.S. Dingus, D.R. Curren, A.A. Oraevsky and S.L. Jacques, "Microscopic spallation process and its potential role in laser tissue ablation", Proceedings of Laser-Tissue Interaction V, Vol. 2134, pp. 434-445, 1994.

7. D.R. Curren and L. Seaman, "Dynamic Failure of Solids," Physics Reports, Vol. 147, pp. 253-388, 1987. 